\title{
Évolution à long terme du statut carboné du sol en monoculture non irriguée du maïs (Zea mays $L$ )
}

\author{
D Plénet, E Lubet, C Juste \\ INRA, station d'Agronomie, centre de recherches de Bordeaux, BP 81, F33883 Villenave-d'Ornon cedex, France
}

(Reçu le 15 octobre 1992; accepté le 6 juillet 1993)

\begin{abstract}
Résumé - Dans 2 dispositifs expérimentaux situés sur limons sableux des Landes de Gascogne, on a suivi pendant respectivement 22 et 25 années l'évolution du statut carboné du sol, en monoculture de maïs avec (TR) et sans (TE) restitution des résidus de culture, en monoculture de maïs avec restitution des résidus de culture suivie par une interculture de ray-grass $(R G)$ et dans un cycle de successions comprenant 4 années de culture de mais avec restitution des résidus de récolte suivies par 3 années de prairie temporaire (PT). Quel que soit le traitement, on observe une décroissance de la teneur en carbone qui, dans le traitement TR, passe en 22 ans de 8,30 à $6,92 \mathrm{~g} / \mathrm{kg}$ (32 à 26 t/ha de C) dans l'un des dispositifs (fig 3) et de 15,50 à $9,65 \mathrm{~g} / \mathrm{kg}$ en 25 ans (57 à 36 t/ha de C) dans l'autre (fig 4). Les différentiels de stocks carbonés induits par les régimes distincts de restitution organique sont peu modifiés au cours du temps par rapport au traitement TR (tableau I) : I'exportation des résidus de culture entraîne un déficit de 3 et 4 tha de $C$ alors que l'introduction de l'engrais vert ou de la prairie temporaire accroît le stock respectivement de 2 et 3 tha et de 2 et 4 t/ha. La mise en place d'une jachère cultivée se traduit en revanche par un déficit du stock atteignant $7 \mathrm{t} /$ ha après respectivement 8 et 11 ans. L'utilisation d'un ajustement exponentiel asymptotique permet de pronostiquer un niveau d'équilibre du carbone à long terme de 6,1 et 8,4 $\mathrm{g} / \mathrm{kg}$ (23 et $31 \mathrm{t} / \mathrm{ha}$ de C) dans les parcelles soumises à la monoculture avec restitution des résidus. La relation de Hénin et Dupuis, établie à partir de coefficients déterminés dans les dispositifs $\left(k_{1}=5,6\right.$ à $6,5 \%-k_{2}=1,16$ et $\left.1,63 \%\right)$, conduit à des teneurs d'équilibre plus faibles $(2,2$ et $3,3 \mathrm{~g} /$ $\mathrm{kg}$ soit 8 et 13 t/ha de C). La valeur approchée des coefficients, dont la détermination sur le terrain ignore l'influence exercée sur l'activité biologique du sol par les variables expérimentales mises en jeu (itinéraires techniques, régimes de restitution), et surtout le caractère trop simplificateur du modèle peuvent être à l'origine de cette sous-estimation.
\end{abstract}

maïs / engrais vert / interculture / jachère / matière organique du sol / résidus de culture

Summary - Change in the organic carbon content of soil in a long-term continuous non-irrigated maize crop. In 2 long-term experiments located on loamy sandy soils in southwest France, change in soil carbon status was measured over a 22- and 25-yr period respectively. Soil cropping was as follows: continuous maize crop with stalks returned to the soil (TR), continuous maize crop with stalks removed (TE), continuous maize crop including a winter annual cover as rye-grass (RG) and rotation involving temporary grassland for 3 yr followed by a maize crop for 4 yr (PT). Whatever the treatment applied, the soil organic carbon content decreased during the course of experiment. In the plots under continuous maize cropping with returned stalks, soil carbon concentration decreased from 8.30 to $6.92 \mathrm{~g} / \mathrm{kg}$ (32 to 26 t organic $C$ ha $^{-1}$ ) over $22 \mathrm{yr}$ and from 15.50 to $9.65 \mathrm{~g} / \mathrm{kg}\left(57\right.$ to $36 \mathrm{t} C \mathrm{ha}^{-1}$ ) over $25 \mathrm{yr}$ respectively in both experiments. No marked change in carbon balance due to the various organic matter treatments occurred during the course of experiment. The amount of stored $C$ decreased by 3 and $4 t$ organic $C$ ha $^{-1}$ respectively in both experiments as a result of continuous removal of plant residues. On the other hand, using a winter cover crop of rye-grass or the establishment of a temporary grassland resulted in an increase of $C$ storage ranging from 2 to 3 tha-1 or from 2 to 4 tha-1 organic $C$. On the contrary, introducing a cultivated fallow in 1980 resulted in a marked decay of organic matter averaging $7 \mathrm{t} \mathrm{ha-1} C$ for both experiments in 1988 and in 1991 respectively. An asymptotic exponential model led to the prediction that the ploughed soil layer would contain 23 and $31 \mathrm{tha}^{-1}$ organic $C(6.1$ and $8.4 \mathrm{~g} / \mathrm{kg} \mathrm{C} \mathrm{content)} \mathrm{at} \mathrm{the}$ equilibrium stage. Using the Henin-Dupuis relationship, which involves parameters based on field measurements $\left(k_{1}\right.$ $=5.6$ and $6.5 \% ; k_{2}=1.16$ and $1.63 \%$ ), calculated equilibrium values of soil carbon level content are much lower. Taking into account data from experiments carried out in other countries, such low values are questionable. The unreliability of the Henin-Dupuis (1945) model could be attributable to its single-compartmental conception and to an un- 
satisfactory approach using the $k_{1}$ and $k_{2}$ parameters calculation. Indeed, such a calculation does not take into account the marked change in soil mineralization due to the various cultural practices or different organic matter fluxes entering the soil in the field experiment that result in a systematic underestimation of predicted equilibrium values.

maize / green manure / cover crop / fallow / soil organic matter / crop residue

\section{INTRODUCTION}

Dans une précédente publication (Lubet et al, 1993) résumant les résultats de 2 expérimentations conduites pendant respectivement 22 et 25 années sur sol sablo-limoneux du Sud Ouest atlantique français, on a montré que la monoculture non irriguée du maïs, système de culture dominant dans la région, n'entraîne pas une baisse des rendements, à cette échelle de temps. Tout au contraire, on observe une augmentation continue de ces derniers, de l'ordre de $1 \mathrm{q} / \mathrm{ha} / \mathrm{an}$, vraisemblablement attribuable au progrès génétique.

Cette étude a par ailleurs montré que l'enlèvement systématique des parties aériennes est sans effet significatif sur les rendements. En revanche, l'introduction d'une inter-culture hivernale d'engrais vert (ray-grass d'italie) ou d'une prairie temporaire de 3 ans suivie par 4 ans de monoculture de maïs provoque, pour l'ensemble de la période expérimentale considérée, un accroissement moyen des rendements de l'ordre de 6 à $8 \%$.

Cette note a pour objectif de présenter les résultats de l'étude de l'évolution du statut carboné des 2 dispositifs, évolution induite par les différents régimes de restitutions organiques mis en œuvre pendant respectivement 22 et 25 années.

\section{MATÉRIEL ET MÉTHODES}

\section{Dispositifs}

Les caractéristiques de ces 2 dispositifs expérimentaux ont été décrites en détail dans une publication précédente (Lubet et al, 1993). Situés à proximité l'un de l'autre, dans la petite région agricole de Chalosse du département des Landes, ils ont été mis en place tous les deux en 1967. Le premier (dispositif de Serreslous) a fait l'objet d'un suivi annuel des rendements jusqu'en 1988 inclus, alors que le second (dispositif de Doazit) a fait l'objet des contrôles jusqu'en 1991 inclus. Une histoire culturale distincte avant la mise en place des expérimentations est à l'origine des teneurs initiales en matière organique très différentes (respec- tivement 1,5 et $2,8 \%$ à Serreslous et Doazit). En outre, la situation topographique de ce dernier et la moins grande épaisseur du substrat sur lequel il repose le rendent plus sensible aux périodes de déficit hydrique estival.

\section{Protocole}

Chacun des 2 dispositifs de type bloc comporte les 5 traitements suivants répétés 4 fois :

- i) traitement TR = monoculture de maîs dont la totalité des résidus de culture est incorporée au sol ;

- ii) traitement TE = monoculture de maïs dont la totalité des parties aériennes est exportée ;

- iii) traitement $\mathrm{RG}=$ monoculture de maïs dont les résidus de récolte sont restitués, suivie d'un engrais vert (ray-grass d'Italie) semé en août et enfoui au printemps avant le semis du maïs suivant ;

- iv) traitement PT = culture de maïs durant 4 années, suivie d'une prairie temporaire de 3 ans. Au cours de l'expérience, 3 prairies ont ainsi été installées : 1968 à 1970,1975 à 1977,1982 à 1984 ;

- v) traitement JA = jachère nue travaillée. Elle a été mise en place en 1980 sur des parcelles portant, depuis le début de l'expérience, une monoculture de mais avec restitution des résidus de récolte. La jachère est traitée de manière strictement analogue aux parcelles portant du maïs pour ce qui concerne toutes les façons culturales ainsi que les apports de fertilisants, d'amendements calcaires et de désherbants, avec cependant un apport supplémentaire de ces derniers à base de glyphosate pour assurer la complète maîtrise des adventices.

La dimension des parcelles élémentaires est de $168 \mathrm{~m}^{2}(30 \times 5,6 \mathrm{~m})$ dans le dispositif de Doazit et de $190 \mathrm{~m}^{2}(34 \times 5,6 \mathrm{~m})$ dans celui de Serreslous.

Les itinéraires techniques mis en œuvre pour la conduite de ces productions ont été décrits en détail dans une publication précédente (Lubet et al, 1993).

\section{Déterminations réalisées}

\section{Évaluation de la production des résidus de récolte}

Seules les parties aériennes comprenant les tiges, les feuilles et les spathes ont donné lieu chaque année à cette évaluation qui a été faite, tout de suite après la 
récolte du grain, par pesée d'un échantillon moyen constitué par des plantes prélevées au hasard dans le traitement TR sur 4 blocs. Après détermination de la quantité de matière sèche des parties aériennes, une aliquote a été prélevée pour déterminer la teneur en carbone du matériel végétal.

\section{Caractéristiques du sol}

À des intervalles de temps variables, précisés dans le tableau I, des échantillons de sol ont été prélevés dans l'ensemble des parcelles de tous les traitements: cette opération a été effectuée au début de l'hiver, par constitution d'un échantillon provenant du mélange de 6 carottages réalisés dans la couche labourée de chacune des parcelles $(0-25 \mathrm{~cm}$ ou $0-23 \mathrm{~cm}$ selon les dispositifs). On a prélevé également dans ces dernières un échantillon du sous-sol $(23-40 \mathrm{~cm}$ ou $25-50 \mathrm{~cm})$.
Huit campagnes de prélèvement complètes ont ainsi été effectuées de 1967 à 1988 dans le dispositif de Serreslous et 7, de 1967 à 1991, dans le dispositif de Doazit.

Le carbone total du sol et celui du matériel végétal ont été dosés par oxydation sulfo-chromique (Anne, 1945) par le laboratoire d'analyse des sols de l'INRA à Arras et le laboratoire d'analyses végétales de I'INRA à Bordeaux. Une grande partie des échantillons stockés après une première analyse ont été repris en fin d'expérience et analysés à nouveau simultanément afin de se soustraire à un biais attribuable à une éventuelle dérive analytique. Pour lever toute ambiguité attachée au mode d'expression du flux de matières organiques de compositions différentes (Boiffin et al, 1986), toutes les variations de teneurs en matière organique du sol ainsi que les entrées sous forme de résidus végétaux ont été exprimées en carbone.

Tableau I. Évolution de la teneur et du stock de carbone du sol.

\begin{tabular}{|c|c|c|c|c|c|c|c|}
\hline & \multicolumn{5}{|c|}{ Teneur en carbone $(\mathrm{g} / \mathrm{kg})$} & \multicolumn{2}{|c|}{ Statistiques } \\
\hline & $T E$ & $T R$ & $R G$ & $P T$ & $J A^{1}$ & $C V$ & Sign \\
\hline \multicolumn{8}{|l|}{ Serreslous } \\
\hline 1967 & & $8,30(32)$ & & & & & \\
\hline 1970 & $7,39^{c}$ & $7,78 \mathrm{bc}$ & $8,35^{b}$ & $10,15^{a}$ & & 4,7 & $\star \star \star$ \\
\hline 1973 & $7,08^{c}$ & $7,50^{c}$ & $7,96^{b}$ & $8,53^{a}$ & & 3,5 & $\star \star \star$ \\
\hline 1974 & $7,22 \mathrm{~b}$ & $7,78 a b$ & $8,40^{a}$ & $8,44^{a}$ & & 6,5 & * \\
\hline 1977 & $7,01^{\mathrm{c}}$ & $7,69 \mathrm{~b}$ & $8,13 a b$ & $8,74^{a}$ & & 5,3 & $\star \star *$ \\
\hline 1978 & $6,63^{d}$ & $7,21 \mathrm{c}$ & 7,67 b & 8,26 a & & 3,8 & 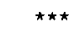 \\
\hline 1982 & $6,49^{c}$ & $6,94^{b}$ & $7,85^{a}$ & $7,78^{a}$ & 7,03 & 3,0 & $\star * *$ \\
\hline 1985 & $6,13^{c}$ & $7,01^{b}$ & $7,54 a b$ & 7,89 a & 5,94 & 5,4 & $\star \star \star \star$ \\
\hline 1988 & $6,08^{d}$ & $6,92^{c}$ & $7,60^{b}$ & $7,92^{a}$ & 5,12 & 2,0 & $* * *$ \\
\hline t/ha & (23) & (26) & (29) & (30) & $(19)$ & & \\
\hline Analyse pluriann ${ }^{2}$ & $6,75^{d}$ & $7,35 \mathrm{c}$ & $7,94^{b}$ & 8,46 a & & 4,5 & $\star \star \star$ \\
\hline \multicolumn{8}{|l|}{ Doazit } \\
\hline 1967 & & $15,50(57)$ & & & & & \\
\hline 1970 & $14,10^{b}$ & $14,02^{b}$ & $14,52 a b$ & $15,33^{a}$ & & 3,5 & * \\
\hline 1974 & $12,20^{c}$ & $12,45 \mathrm{bc}$ & $13,15 a b$ & $13,40^{a}$ & & 3,6 & * \\
\hline 1978 & $10,88^{b}$ & $11,35^{b}$ & $12,18^{a}$ & $12,90^{a}$ & & 4,1 & $\star \star$ \\
\hline 1982 & $10,30^{c}$ & $10,76 \mathrm{bc}$ & 11,54 ab & $12,35^{a}$ & 10,76 & 4,5 & $\star \star \star$ \\
\hline 1985 & $9,95^{b}$ & $10,38^{b}$ & $11,00 \mathrm{ab}$ & $11,65^{a}$ & 9,51 & 5,8 & * \\
\hline 1988 & $9,33^{b}$ & $9,95 a b$ & $10,50^{a}$ & $10,65^{a}$ & 8,71 & 5,0 & * \\
\hline 1991 & $8,76^{c}$ & $9,65 \mathrm{~b}$ & $10,20^{a}$ & 10,19 a & 7,84 & 2,4 & $\star \star \star \star$ \\
\hline tha & $(32)$ & $(36)$ & $(38)$ & $(38)$ & (29) & & \\
\hline Analyse pluriann ${ }^{2}$ & $10,79^{d}$ & $11,22^{c}$ & $11,87^{b}$ & $12,35^{a}$ & & 4,4 & $\star \star \star$ \\
\hline
\end{tabular}

Entre parenthèses : carbone, exprimé en tha ; ${ }^{1}$ La jachère n'est pas prise en compte pour les analyses de variance. ${ }^{2}$ Les moyennes pluriannuelles des traitements n'intègrent pas l'année 1967. Sur une ligne, les valeurs suivies d'une même lettre ne sont pas significativement différentes au seuil de $5 \%$ (test de Newman-Keuls). CV: coefficient de variation ; Sign : interprétation statistique de l'analyse de variance ; ${ }^{\star}$ : significatif au seuil de $5 \% ;{ }^{\star \star}$ : significatif au seuil de $1 \% ;{ }^{\star \star \star}$ : significatif au seuil de $1 \%$. 


\section{Traitement statistique des résultats}

Les moyennes par traitement des données obtenues chaque année ont fait l'objet d'une analyse de variance année par année et d'une analyse pluriannuelle pour comparer les effets moyens des traitements. Lorsque le test de Fisher est significatif au seuil de $5 \%$, une comparaison des moyennes est réalisée avec le test de Newman-Keuls au seuil de 5\%. Les cinétiques d'évolution du carbone du sol ont été ajustées par la méthode des moindres carrés dans des modèles de régression non-linéaire. Une observation détaillée de la répartition des résidus ainsi que l'analyse des écart-types approchés des différents estimateurs et des matrices de corrélation entre les paramètres a permis de retenir les modèles les plus cohérents. Tous les ajustements ont été réalisés en prenant en compte la moyenne de chaque traitement.

\section{RÉSULTATS}

\section{Effet des traitements sur la teneur en carbone des parcelles au terme de l'expérience}

L'analyse des résultats obtenus (tableau I) montre que, dans tous les cas de figure, il y a diminution du stock organique du sol depuis la mise en place de l'expérimentation en 1967. Cette diminution a été plus marquée au cours des premières années dans le dispositif Doazit dont l'histoire culturale explique la plus grande richesse initiale en carbone.

Ces résultats font apparaître aussi une différenciation significative des teneurs en carbone des parcelles soumises à des régimes distincts de restitutions organiques: comme on pouvait le prévoir, l'exportation des résidus de récolte conduit aux teneurs en carbone les plus faibles (92 à $95 \%$ de la teneur en $\mathrm{C}$ caractérisant le traitement avec incorporation des résidus), alors que l'introduction de la prairie (112 à $115 \%$ ) ou de l'inter-culture d'engrais vert (107 à 108\%) améliore significativement le statut carboné des parcelles.

Si pour la totalité de la durée de l'expérimentation on aboutit, en se référant à l'interprétation pluriannuelle, à un classement identique de l'effet des traitements sur le statut carboné dans les 2 dispositifs, la différenciation est cependant beaucoup moins nette dans le dispositif Doazit. Par exemple, on enregistre annuellement dans ce site une simple tendance à un enrichissement en carbone des parcelles avec restitution de ré- sidus par rapport aux parcelles sans restitution ; seule l'analyse pluriannuelle des résultats permet de faire apparaître un effet significatif. II en va de même de l'écart séparant les traitements ray-grass et prairie temporaire, l'effet de ce dernier se manifestant encore en 1984, 7 années après le dernier retournement de la prairie. Par contre dans le dispositif Serreslous, la différenciation du statut carboné des parcelles en fonction des traitements se manifeste plus nettement, l'année 1988 conduisant au même classement (statistiquement significatif) que celui fourni par l'analyse pluriannuelle.

La figure 1 montre que l'introduction, pourtant tardive, de la jachère accélère considérablement la perte de carbone ; en effet, après respectivement 8 et 11 ans d'application de ce traitement du sol, la teneur en $\mathrm{C}$ est inférieure de $26 \%$ à celle mesurée dans les parcelles en maïs avec restitution des résidus dans le dispositif Serreslous, et de $19 \%$ dans le dispositif Doazit.

\section{Cinétique de décroissance de la teneur en carbone du sol}

Pour décrire les cinétiques de décroissance des teneurs en carbone des sols, 2 démarches ont été utilisées. La première consiste à essayer de valider le modèle de Hénin et Dupuis (1945). La deuxième approche vise à rechercher, a posteriori, un modèle mathématique permettant le meilleur ajustement possible des points expérimentaux afin de pouvoir pronostiquer les taux de carbone à l'équilibre.

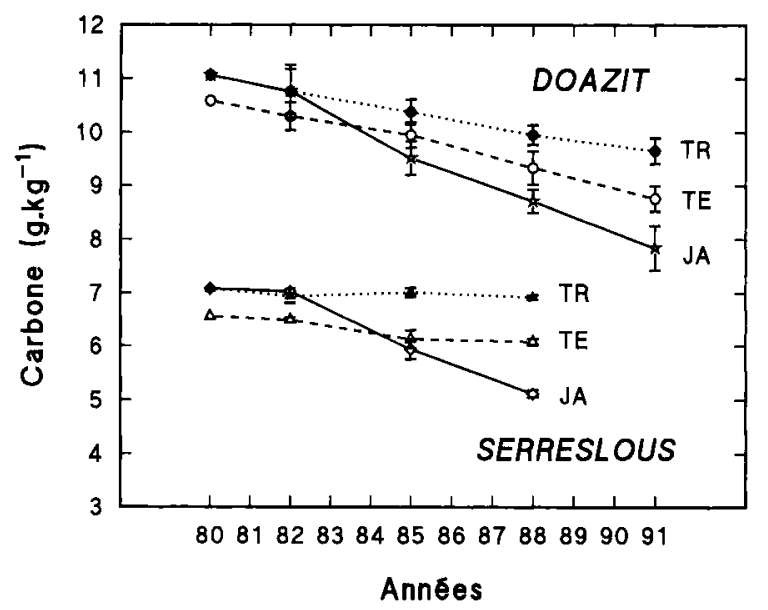

Fig 1. Évolution de la teneur en carbone dans les parcelles en jachère nue travaillée (JA), en monoculture de maïs avec restitution des résidus de culture (TR) et en monoculture de maïs avec exportation des résidus de culture (TE) sur les 2 sites. Les barres verticales représentent \pm l'écart type. 


\section{Description de la cinétique selon le modèle de Hénin et Dupuis}

Ce modèle fait intervenir 2 paramètres, assimilables sous certaines conditions, à des coefficients annuels moyens : le coefficient $k_{2}$, qui correspond à un taux de destruction annuel moyen du carbone du sol, et le coefficient $k_{1}$ (coefficient isohumique), qui traduit un rendement en carbone des résidus de récolte que l'on enfouit dans le sol.

Sa formulation générale est la suivante :

$$
\Delta C=k_{1} A-k_{2} C
$$

avec : $A=$ apport annuel de résidus de récolte ( $\mathrm{g} /$ $\mathrm{kg}$ de terre fine) ; $C=$ teneur en carbone du sol ( $\mathrm{g} / \mathrm{kg}$ de terre fine) $\Delta C=$ variation de la teneur en carbone du sol.

Pour un intervalle d'intégration suffisamment grand devant le pas de mesure, l'intégration de l'expression ci-dessous donne:

$$
\mathrm{C}=\left(C_{0}-\frac{k_{1} \mathrm{~A}}{k_{2}}\right) e^{-k_{2} \mathrm{t}}+\frac{k_{1} \mathrm{~A}}{k_{2}}
$$

avec $C_{0}=$ teneur en carbone du sol au temps $t_{0}$ ( $\mathrm{g} / \mathrm{kg}$ de terre fine) ; $t=$ temps (année).

Ce qui permet d'estimer à :

$$
\frac{k_{1} \times A}{k_{2}}
$$

le taux d'équilibre à long terme de la teneur en carbone du sol pour un régime donné de restitution.

En se fondant sur la formulation discrète du phénomène préconisée par Chadœuf et al (1984), on a estimé conjointement, par la méthode des moindres carrés, les paramètres $k_{1}, k_{2}$ et $C_{0}$. Les apports annuels de résidus de récolte (A) pris en compte pour le calcul figurent dans le tableau II.

Les résultats de ce calcul (tableau III) confirment ceux des auteurs ci-dessus, à savoir la grande difficulté à estimer valablement $k_{1}$ et $k_{2}$ par une démarche de ce type, en raison, d'une part, de la très grande variance dont ils sont affectés pour le temps d'expérimentation considéré et, d'autre part, de leur extrême sensibilité au moindre changement de la période prise en
Tableau II. Apports de carbone par les résidus de récolte (parties aériennes) du maïs ( \pm écart type).

\begin{tabular}{lcc}
\hline & $\begin{array}{c}\text { Essai } \\
\text { Serreslous }\end{array}$ & $\begin{array}{c}\text { Essai } \\
\text { Doazit }\end{array}$ \\
\hline $\begin{array}{l}\text { Apport } \\
\text { (kg/ha/an matière sèche) }\end{array}$ & $5263 \pm 741$ & $5376 \pm 688$ \\
$\begin{array}{l}\text { Teneur en carbone } \\
\text { (\% matière sèche) }\end{array}$ & $42,3 \pm 1,6$ & $43,5 \pm 1,3$ \\
Carbone (kg/ha/an) & $2226 \pm 341$ & $2339 \pm 283$ \\
\hline
\end{tabular}

compte pour l'ajustement. Par ailleurs, la très forte corrélation qui lie entre eux ces 2 coefficients traduit leur dépendance et remet donc en cause la pertinence même du modèle.

On a donc cherché à estimer séparément $k_{1}$ et $k_{2}$ à partir de l'information disponible dans les parcelles du dispositif pour lesquelles la restitution des résidus est partielle (traitement TE), ou nulle (traitement JA).

\section{Coefficient $\mathrm{k}_{2}$}

II a été déterminé en utilisant les données fournies par les parcelles du traitement TE (totalité des parties aériennes exportées) pour lesquelles on considère que $A=0$ (relation 1 ). En fait il s'agit d'un coefficient $k_{2}$ apparent puisque ces parcelles bénéficient chaque année des «entrées» de carbone que représentent les racines.

Les paramètres des ajustements ainsi réalisés sont rassemblés dans le tableau IV. Pour le dispositif de Serreslous, on obtient un bon ajustement des données ainsi qu'une distribution aléatoire des résidus en prenant comme année de départ 1970. Dans le cas du dispositif Doazit, la répartition non aléatoire des résidus a conduit à scinder la durée de l'expérience en 2 périodes: 1967-1978 et 1978-1991, cette dernière reflétant l'instauration d'une cinétique de dégradation de la matière organique plus conforme au régime actuel des restitutions (fig 2).

On a aussi tenté de rendre compte du comportement particulier du dispositif Doazit, lié à sa plus forte teneur initiale en matière organique, en ajustant les points expérimentaux selon un modèle bicompartimental, somme de 2 exponentielles, pour tenir compte de l'existence 
Tableau III. Estimation simultanée des 3 paramètres de l'ajustement au modèle d'évolution du carbone de Hénin et Dupuis (1945) dans les parcelles où les résidus de récolte sont enfouis (TR).

\begin{tabular}{|c|c|c|c|c|c|c|c|}
\hline Dispositif & $c_{0}$ & $k_{1}$ & $\mathrm{k}_{2}$ & $S C R$ & $R^{2}$ & $d l$ & $r\left(\mathrm{k}_{1}, \mathrm{k}_{2}\right)$ \\
\hline \multicolumn{8}{|l|}{ Serreslous } \\
\hline$(1970-1988)$ & $\begin{array}{r}7,83 \\
( \pm 0,18)\end{array}$ & $\begin{array}{r}0,099 \\
( \pm 1,090)\end{array}$ & $\begin{array}{r}0,015 \\
( \pm 0,087)\end{array}$ & 0,207 & 0,793 & 5 & 0,999 \\
\hline$(1967-1988)$ & $\begin{array}{c}8,26 \\
( \pm 0,18)\end{array}$ & $\begin{array}{c}0,460 \\
( \pm 0,550)\end{array}$ & $\begin{array}{c}0,044 \\
( \pm 0,043)\end{array}$ & 0,216 & 0,879 & 6 & 0,999 \\
\hline \multicolumn{8}{|l|}{ Doazit } \\
\hline$(1967-1991)$ & $\begin{array}{c}15,56 \\
( \pm 0,18)\end{array}$ & $\begin{array}{c}0,900 \\
( \pm 0,120)\end{array}$ & $\begin{array}{c}0,068 \\
( \pm 0,007)\end{array}$ & 0,076 & 0,998 & 5 & 0,997 \\
\hline$(1978-1991)$ & $\begin{array}{c}11,34 \\
( \pm 0,03)\end{array}$ & $\begin{array}{c}0,221 \\
( \pm 0,180)\end{array}$ & $\begin{array}{r}0,026 \\
( \pm 0,011)\end{array}$ & 0,002 & 0,999 & 2 & 0,999 \\
\hline
\end{tabular}

$\mathrm{C}_{0}$ : teneur initiale en carbone $(\mathrm{g} / \mathrm{kg})$ du sol pour la période servant à l'ajustement ; SCR : somme des carrés des résidus ; $( \pm)$ : écart type ; $\mathrm{dl}$ : degré de liberté $; \mathrm{r}\left(k_{1}, k_{2}\right)$ : coefficient de corrélation linéaire entre les coefficients $k_{1}$ et $k_{2} ; \mathrm{R}^{2}:$ partie de la variance expliquée par le modèle

éventuelle de 2 compartiments de carbone possédant des demi-vies différentes:

$$
C(g / k g)=A e^{\alpha t}+B e^{\beta t}
$$

avec : $A=9,26( \pm 19,68) ; B=6,35( \pm 19,77)$ $\alpha=-0,057( \pm 0,091) ; \beta=0,0019( \pm 0,067) ; R^{2}$ $=0,993$.

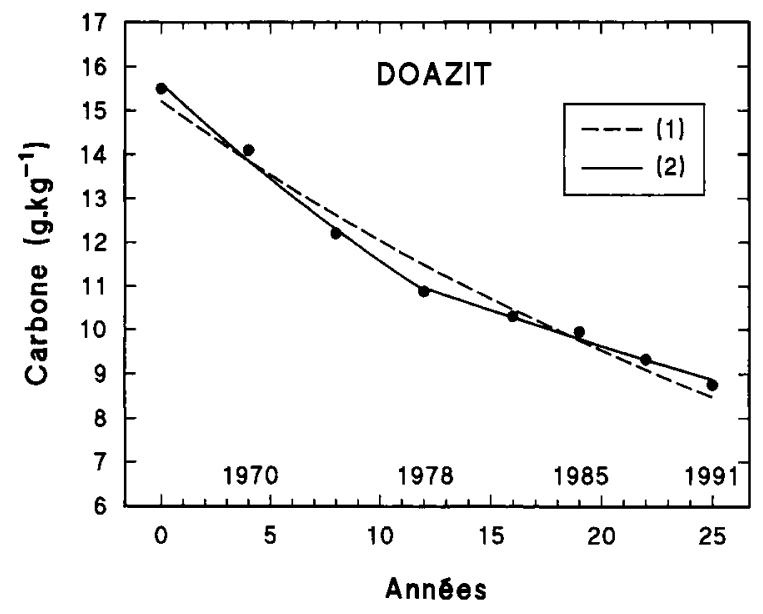

Fig 2. Modélisation de la cinétique du carbone selon le modèle de Hénin et Dupuis (1945) dans les parcelles en monoculture de maïs avec exportation des résidus de culture dans le dispositif de Doazit. (1) : modélisation relative à la période 1967-1991 ; (2) : modélisation relative aux périodes 19671978 et $1978-1991$.
Tableau IV. Paramètres de l'ajustement au modèle d'évolution du carbone de Hénin et Dupuis (1945) dans les parcelles d'où les résidus de récolte sont exportés (TE).

\begin{tabular}{llllll}
\hline Dispositif & $C_{0}$ & $\mathrm{k}_{2}$ & SCR & $R^{2}$ & $d l$ \\
\hline
\end{tabular}

Serreslous

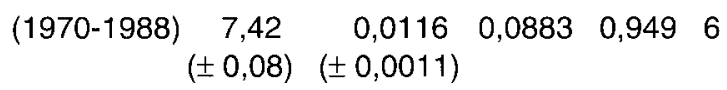

Doazit

$\begin{array}{llllll}(1967-1991) & 15,21 & 0,0234 & 0,8890 & 0,978 & 6\end{array}$ $( \pm 0,28) \quad( \pm 0,0015)$

$\begin{array}{llllll}(1967-1978) & 15,62 & 0,0299 & 0,0827 & 0,993 & 2\end{array}$ $( \pm 0,18) \quad( \pm 0,0018)$

$\begin{array}{llllll}(1978-1991) & 10,97 & 0,0163 & 0,0485 & 0,982 & 3\end{array}$ $( \pm 0,11) \quad( \pm 0,0013)$

$\mathrm{C}_{0}$ : teneur initiale en carbone $(\mathrm{g} / \mathrm{kg})$ du sol pour la période servant à l'ajustement ; SCR : somme des carrés des résidus ; $(t)$ : écart type ; $\mathrm{dl}$ : degré de liberté ; $\mathbf{R}^{2}$ : partie de la variance expliquée par le modèle. 
Malgré un ajustement correct des valeurs mesurées, les écart-types considérables affectant l'ensemble des paramètres, les très forts coefficients de corrélation liant les estimations et la très faible valeur du coefficient de l'une des exponentielles $(0,0019$ - qui n'est pas statistiquement différent de zéro) ne nous permettent pas de retenir ce modèle. Ces résultats semblent indiquer toutefois qu'une exponentielle asymptotique pourrait être utilisée pour expliquer la cinétique de décroissance du carbone.

\section{Coefficient $\mathrm{k}_{1}$}

Il a été déterminé en comparant l'évolution de la teneur en carbone des parcelles soumises aux différents régimes de restitution des résidus.

\section{Parties aériennes}

L'enrichissement en matière organique, dû à l'enfouissement des parties aériennes, est calculé à partir de la différence des teneurs en carbone des parcelles dans lesquelles on incorpore (traitement TR) ou non (traitement TE) les résidus.

Le poids moyen de matière sèche des parties aériennes résiduelles mesuré chaque année après récolte ainsi que leur teneur en carbone sont indiqués dans le tableau II. Leur contribution à l'accroissement de la matière organique du sol est évaluée en effectuant le rapport du $C$ total ajouté par leur intermédiaire à l'enrichissement en cet élément effectivement constaté dans un intervalle de temps donné. Dans le cas du dispositif de Serreslous et pour la période comprise entre 1967 et 1988, soit après 22 ans de gestion différenciée des résidus de récolte, ce rapport a pu être ainsi évalué à 6,52\% (tableau V). Dans le dispositif Doazit, après 25 années de différenciation, on obtient pour $k_{1}$ une valeur de $5,63 \%$. Si l'on prend en compte le sol et le sous-sol (profondeur totale $0-40$ ou $0-50 \mathrm{~cm}$ ), les valeurs de ces coefficients s'élèvent respectivement, pour les dispositifs Serreslous et Doazit, à $8,23 \%$ et $7,66 \%$. Elles se situent indiscutablement dans la partie inférieure de l'éventail ( 6 à $30 \%$ ) des coefficients relatifs aux parties aériennes des céréales à paille et du maïs cités par un certain nombre d'auteurs (Rémy et Marin-Laflèche, 1976 ; Delphin et Conesa, 1979 ; Lubet et Juste, 1979 ; Müller, 1982 ; Delas et Molot, 1983 ; Picard, 1983 ; Guerif, 1987).

\section{Parties souterraines}

On a essayé d'approcher la contribution de la production racinaire (racines et exsudats) en comparant, en fin d'expérience, la teneur en car-
Tableau V. Évaluation du coefficient isohumique $\left(K_{1}\right)$ des résidus de récolte (parties aériennes) du maïs.

\begin{tabular}{lccl}
\hline $\begin{array}{l}\text { Période prise } \\
\text { en compte }\end{array}$ & $\begin{array}{c}\text { Nombre } \\
\text { d'années }\end{array}$ & \multicolumn{2}{c}{$\mathrm{K}_{1} \%^{*}$} \\
& & $\begin{array}{c}\text { Essai de } \\
\text { Serreslous }\end{array}$ & $\begin{array}{c}\text { Essai de } \\
\text { Doazit }\end{array}$ \\
& & & \\
\hline & & 16,64 & - \\
$1967-1970$ & 4 & 10,24 & - \\
$1967-1973$ & 7 & 11,95 & 4,94 \\
$1967-1974$ & 8 & 10,55 & - \\
$1967-1977$ & 11 & 8,25 & 6,20 \\
$1967-1978$ & 12 & 4,80 & 4,55 \\
$1967-1982$ & 16 & 7,91 & 3,58 \\
$1967-1985$ & 19 & 6,52 & 4,46 \\
$1967-1988$ & 22 & - & 5,63 \\
$1967-1991$ & 25 & $8,23^{\star \star}$ & - \\
& & - & $7,66^{\star \star}$ \\
$1967-1988$ & 22 & & \\
$1967-1991$ & 25 & & \\
\hline
\end{tabular}

* : valeurs calculées en tenant compte de l'enrichissement en carbone du sol; ${ }^{* *}$ : valeur calculée en tenant compte de l'enrichissement en carbone du sol et du sous-sol.

bone des parcelles conduites en jachère travaillée depuis 1980 (traitement $J A$ ) à celle des parcelles où les parties aériennes sont exportées (traitement TE).

Mais, contrairement au cas précédent, on ne dispose pas pour la présente expérimentation de mesures concernant la biomasse racinaire restituée chaque année par la culture de maïs. On peut cependant se référer à la bibliographie (Hénin et Dupuis, 1945 ; Müller, 1982 ; Boiffin et al, 1986 ; Charpentier, 1986 ; Tardieu et Manichon, 1987 ; Balesdent et Balabane, 1992) ou aux résultats obtenus lors d'une recherche antérieure réalisée en conditions très voisines (Lubet et Juste, 1985) et considérer que la biomasse racinaire représente entre $15 \%$ et $20 \%$ de la biomasse aérienne totale en culture non irriguée.

On a indiqué dans le tableau $\mathrm{VI}$ les valeurs des coefficients isohumiques des systèmes racinaires, établies en considérant que la couche labourée renferme environ $85 \%$ du système racinaire (Lubet et Juste, 1985). Les calculs ont été réalisés en prenant pour hypothèses successives une production de racines équivalant à 15 ou à $20 \%$ de la production totale de parties aériennes. On obtient ainsi des coefficients variant de 32 à $60 \%$. Si, tenant compte des hypothèses de Barber et Martin (1976), de Barber (1979) et de Sauerbeck et Johnen (1977), on suppose 
Tableau VI. Estimation du coefficient isohumique $\left(K_{1}\right)$ du système racinaire du maïs.

Variation du stock organique du sol

(TE-JA) - (kg/ha de C)

Biomasse parties aériennes

(kg/ha/an MS)
3648

12400
3404

12220

\begin{tabular}{|c|c|c|c|c|}
\hline & $15 \%$ * & $20 \%$ * & $15 \%$ * & $20 \%$ * \\
\hline $\begin{array}{l}\text { Biomasse racinaire sur } 0-25 \mathrm{~cm} \\
(\mathrm{~kg} / \mathrm{ha} \mathrm{MS})\end{array}$ & 1580 & 2110 & 1558 & 2077 \\
\hline $\begin{array}{l}\text { Carbone racinaire sur } 0-25 \mathrm{~cm} \\
(\mathrm{~kg} / \mathrm{ha} / \text { an de } \mathrm{C})\end{array}$ & 680 & 907 & 670 & 893 \\
\hline Coefficient $K_{1}$ des racines (en $\%$ ) & $60 \%$ & $45 \%$ & $42 \%$ & $32 \%$ \\
\hline $\begin{array}{l}\text { Coefficient } K_{1} \text { des racines } \\
+ \text { exsudats racinaires }{ }^{\star *}\end{array}$ & $30 \%$ & $22,5 \%$ & $21 \%$ & $16 \%$ \\
\hline
\end{tabular}

* : hypothèse de biomasse racinaire : $15 \%$ et $20 \%$ des parties aériennes, dont $85 \%$ dans l'horizon laboure ; ** : exsudats racinaires produits en quantité équivalente à la biomasse racinaire.

que la production d'exsudats racinaires au cours du cycle de développement de la plante est comparable à celle du matériel racinaire, les valeurs du coefficient isohumique caractérisant la totalité de la production racinaire se situent dans un éventail de 16 à $30 \%$.

\section{Comparaison de l'estimation des coefficients $\mathrm{k}_{1}$ par approche expérimentale ou par ajustement}

Comme souligné précédemment, la détermination conjointe de $k_{1}, k_{2}$ et $C_{0}$ par ajustement au modèle de Hénin et Dupuis (1945) conduit, pour $k_{1}$ notamment, à des valeurs non interprétables en terme de rendement en matière organique stable du sol.

On a donc recalculé ce paramètre en attribuant au coefficient $k_{2}$ les valeurs obtenues pour les parcelles du traitement TE. Les résultats de ce calcul sont rassemblés dans le tableau VII. Les valeurs de $k_{1}$ ainsi déterminées (4,3 à 7,6\%) sont dans l'ordre de grandeur de celles fournies par l'approche expérimentale (5,6 à 6,2\%). Elles confirment les observations de Muller et Rémy (1980) et de Djakovitch (1988) montrant que les parties aériennes du maïs ont une plus faible ap- titude à fournir de la matière organique stable au sol que les céréales à paille.

Taux d'équilibre du carbone dans les parcelles où la totalité des résidus de culture est restituée

On admet que cet équilibre est atteint lorsque la matière organique générée annuellement par les résidus de culture compense exactement celle qui disparaît par minéralisation. On a alors :

$$
k_{1} A=k_{2} C
$$

avec $A=$ masse du carbone des résidus enfouis annuellement (en $\mathrm{g} / \mathrm{kg}$ de terre fine) et $C=$ teneur en carbone du sol. On a fait figurer dans le tableau VIII les valeurs des limites vers lesquelles vont tendre ainsi les teneurs en carbone de la couche labourée des deux dispositifs. Ces valeurs $(2,2$ à 3,3 de carbone en $\mathrm{g} / \mathrm{kg}$ de terre fine, soit de 8 à $13 \mathrm{t}$ de carbone/ha de couche labourée) ont été calculées en prenant en compte le coefficient $k_{1}$ évalué à partir de la variation de la teneur en carbone de l'horizon de surface. Par ailleurs, on a fait l'hypothèse que le taux de minéralisation du carbone du sol dans les parcelles 
Tableau VII. Paramètres de l'ajustement au modèle d'évolution de Hénin et Dupuis (1945) dans les parcelles où les résidus de récolte sont enfouis (TR) pour $k_{2}$ fixé.

\begin{tabular}{|c|c|c|c|c|c|c|c|}
\hline Dispositif & $c_{0}$ & $\mathrm{k}_{1}$ & $\mathrm{k}_{2}$ & SCR & $R^{2}$ & $d l$ & $r\left(\mathrm{k}_{1}, C_{0}\right)$ \\
\hline \multicolumn{8}{|l|}{ Serreslous } \\
\hline$(1967-1988)$ & $\begin{array}{r}8,17 \\
( \pm 0,12)\end{array}$ & $\begin{array}{c}0,043 \\
( \pm 0,016)\end{array}$ & 0,0116 & 0,237 & 0,868 & 7 & $-0,821$ \\
\hline$(1970-1988)$ & $\begin{array}{r}7,82 \\
( \pm 0,12)\end{array}$ & $\begin{array}{c}0,053 \\
( \pm 0,019)\end{array}$ & 0,0116 & 0,206 & 0,793 & 6 & $-0,791$ \\
\hline \multicolumn{8}{|l|}{ Doazit } \\
\hline$(1967-1991)$ & $\begin{array}{c}15,08 \\
( \pm 0,26)\end{array}$ & $\begin{array}{c}0,076 \\
( \pm 0,025)\end{array}$ & 0,0234 & 0,817 & 0,973 & 6 & $-0,754$ \\
\hline$(1978-1991)$ & $\begin{array}{c}11,34 \\
( \pm 0,02)\end{array}$ & $\begin{array}{c}0,062 \\
( \pm 0,004)\end{array}$ & 0,0163 & 0,003 & 0,998 & 3 & $-0,799$ \\
\hline
\end{tabular}

$k_{2}$ : valeur des coefficients obtenus par des ajustements sur TE (voir tableau IV) $; \mathrm{C}_{0}:$ teneur initiale en carbone (g/kg) du sol pour la période servant à l'ajustement; SCR : somme des carrés des résidus; $( \pm)$ : écart type ; dl : degré de liberté ; $r\left(k_{1}, \mathrm{C}_{0}\right)$ : coefficient de corrélation linéaire entre le coefficient $k_{1}$ et la teneur initiale en carbone; $\mathrm{R}^{2}$ : partie de la variance expliquée par le modèle.

Tableau VIII. Estimation du niveau d'équilibre du carbone du sol selon le modèle de Hénin et Dupuis (1945) en monoculture de maïs avec restitution des résidus.

Dispositif de Serreslous Dispositif de Doazit

$\begin{array}{lll}K_{2} & 0,0116 & 0,0163 \\ K_{1}{ }^{\star} & 0,0652 & 0,0563 \\ \text { C (\%o) } & 3,29 & 2,18 \\ \text { C (t/ha) } & 13 & 8\end{array}$

* $: k_{1}$ d'après le stock en carbone de la couche labourée.

dont on exporte les résidus est identique à celui des parcelles où on les restitue.

\section{Ajustement de la cinétique selon un modèle exponentiel asymptotique}

Déjà utilisée par Fardeau et al (1988), cette approche consiste à rechercher les paramètres de l'exponentielle de la forme:

$$
C(\mathrm{~g} / \mathrm{kg})=A+B \mathrm{e}^{-k \mathrm{t}}
$$

avec $A=$ valeur d'équilibre de la teneur en carbone du sol, $B$ et $k=$ paramètres.

Cette modélisation fréquemment employée pour décrire la cinétique d'évolution de l'azote total du sol (Jenkinson, 1988) revient à supposer l'existence dans le sol d'un compartiment organique $(A)$ dont la vitesse de minéralisation serait nulle et qui représenterait donc, à la limite, le niveau d'équilibre atteint par la parcelle pour un régime de restitution donné. Ce modèle est une écriture simplifiée du modèle somme de 2 exponentielles [2] en considérant que $\alpha=0$. Le tableau IX présente les valeurs des différents paramètres lorsque ce modèle est ajusté aux teneurs en carbone du sol correspondant aux traitements TE, TR et RG. En outre et comme le laissent apparaître les figures 3 et 4 , les incorporations massives de carbone au moment du retournement des prairies en 1970, 1977 et 1984 n'ont pas permis de parvenir à un ajustement valable pour ce traitement. Globalement, les ajustements obtenus avec ce modèle permettent d'expliquer correctement l'évolution des teneurs en carbone du sol. À noter cependant que, sans doute en raison du nombre insuffisant de données disponibles au cours de la période expérimentale, certains des paramètres, notamment ceux relatifs au traitement engrais vert 
Tableau IX. Estimation du niveau d'équilibre du carbone du sol selon le modèle exponentiel asymptotique.

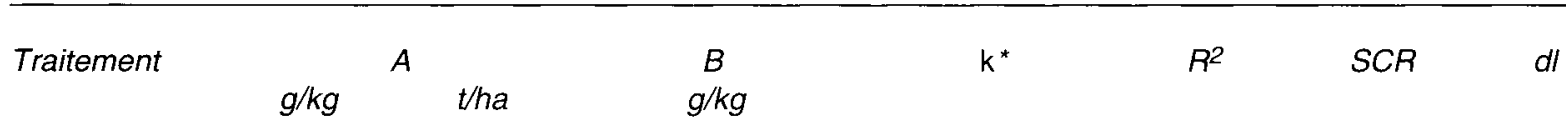

Doazit

(1967-1991)

TE

6,932

(26)

8,686

$( \pm 0,807)$

$0,016)$

0,293

5

TR

8,420

7,157

$( \pm 0,303)$

0,070

0,998

0,076

5

RG

$$
7,175
$$

8,420

$( \pm 0,012)$

$( \pm 0,899)$

$( \pm 0,849)$

0,042
$( \pm 0,007)$

0,997

0,077

5

Serreslous

(1967-1988)

$\begin{array}{cccccccc}\text { TE } & \begin{array}{c}5,352 \\ ( \pm 0,622)\end{array} & (20) & \begin{array}{c}2,858 \\ ( \pm 0,569)\end{array} & \begin{array}{c}0,062 \\ ( \pm 0,025)\end{array} & 0,962 & 0,145 & 6 \\ \text { TR } & \begin{array}{c}6,067 \\ ( \pm 1,373)\end{array} & (23) & \begin{array}{c}2,190 \\ ( \pm 1,288)\end{array} & \begin{array}{c}0,045 \\ ( \pm 0,045)\end{array} & 0,879 & 0,216 & 6 \\ \text { RG } & \begin{array}{r}5,753 \\ ( \pm 9,418)\end{array} & (22) & \begin{array}{c}2,616 \\ ( \pm 9,317)\end{array} & \begin{array}{c}0,018 \\ ( \pm 0,077)\end{array} & 0,791 & 0,143 & 6\end{array}$

TE : monoculture de maïs avec exportation des résidus de culture ; TR : monoculture de maïs avec restitution des résidus de culture ; RG : monoculture de maïs avec restitution des résidus de culture suivie par une culture de ray-grass d'Italie pendant l'interculture ; modèle de régression: $\mathrm{C} g / \mathrm{kg}=\mathrm{A}+\mathrm{B} \mathrm{e}^{-\mathrm{kt}} ; \mathrm{A}$ : teneur en carbone du sol à l'équilibre $(\mathrm{g} / \mathrm{kg}$ de terre fine et en $\mathrm{t} / \mathrm{ha}) ; t:$ temps (années) ; $k$ et $B$ : constantes ; autres symboles : voir légende du tableau VII.

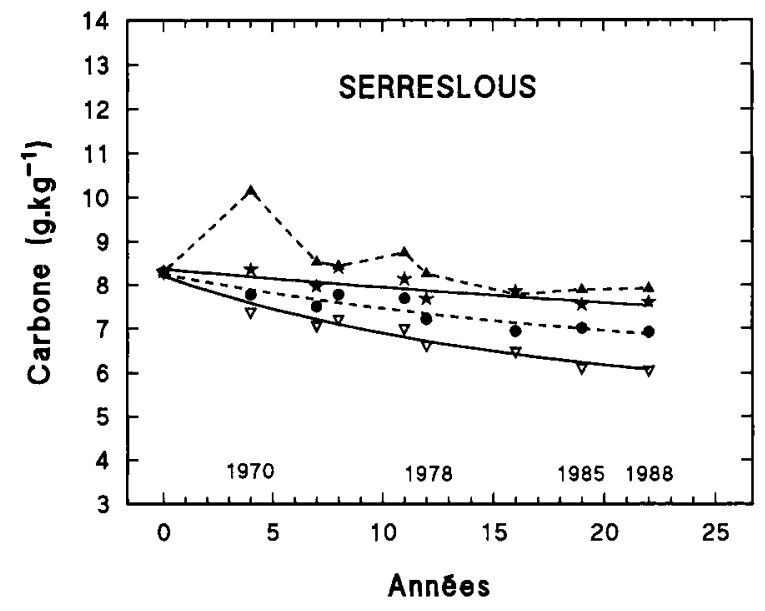

Fig 3. Cinétique du carbone dans le dispositif de Serreslous. $\Delta-\cdots$ PT : prairie temporaire ; avec interculture de ray-grass; - TR : monoculture avec restitution des résidus de culture ; $\nabla----\nabla$ TE: Monoculture avec exportation des résidus de culture. TR, TE, RG : les modèles ajustés aux données expérimentales sont des exponentielles asymptotiques.

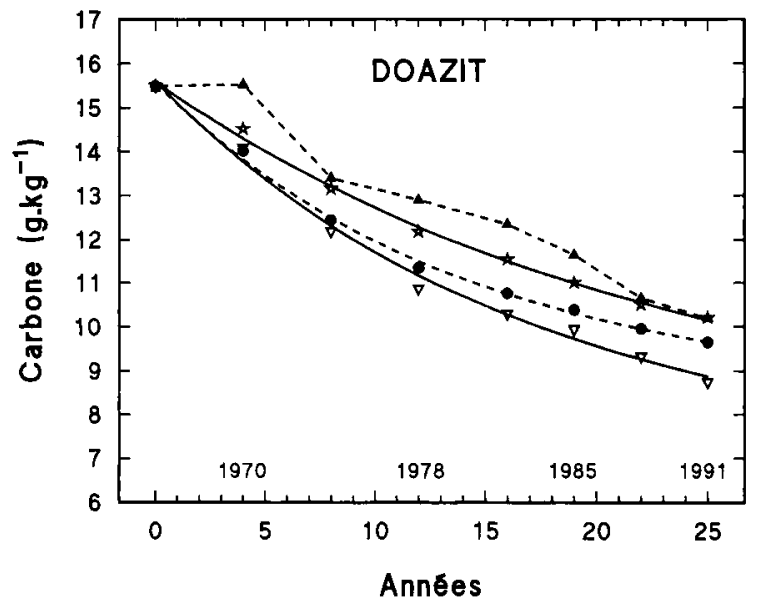

Fig 4. Cinétique de disparition du carbone dans le dispositif de Doazit. $\mathbf{A - - - \Delta}$ PT : prairie temporaire ; ---- TR : monoculture avec restitution des résidus de culture $; \nabla---\nabla$ TE : monoculture avec exportation des résidus de culture ; $R G$ : monoculture avec interculture de ray-grass. TR, TE, $R G$ : les modèles ajustés aux données expérimentales sont des exponentielles asymptotiques. 
(RG) pour le dispositif Serreslous, sont affectés d'écart-types très élevés.

\section{DISCUSSION}

\section{Effet des traitements sur l'évolution du statut carboné du sol à l'échelle de 2 décennies}

L'un des points essentiels à souligner est l'importance de la teneur initiale en carbone pour la mise en évidence de l'effet des régimes de restitution expérimentés. Dans le dispositif Serreslous où la concentration initiale en carbone est faible, la différenciation du statut carboné en fonction du régime de restitution se manifeste dès la première décennie (Lubet et Juste, 1979).

En revanche, dans le dispositif Doazit, dont I'histoire culturale pré-expérimentale est différente (défrichement plus récent, apports importants de fumier, ...), seule l'interprétation statistique pluriannuelle des résultats permet de vérifier le classement attendu de l'impact moyen des différents régimes de restitution :

$$
\mathrm{TE}<\mathrm{TR}<\mathrm{RG}<\mathrm{PT}
$$

Comme déjà souligné par Jenkinson et Rayner (1977) et par Boiffin et al (1986), les résultats de cette étude confirment donc la lenteur du passage d'un équilibre à un autre quand on substitue, à un régime de restitutions organiques pratiqué pendant de nombreuses années, un régime nouveau très différent.

On notera par ailleurs que, par rapport au trajtement de référence TR (monoculture de maïs avec restitution des résidus), les différentiels de stock carboné induits à l'échelle de 2 décennies par les différents régimes de restitution sont dans l'ensemble assez modestes ( 3 et 4 t/ha de déficit dans le cas d'une exportation continue des parties aériennes, 2 à $3 t /$ ha de gain par l'engrais vert et, également, 2 et 4 tha de gain observé 4 et 7 années après le dernier retournement de prairie, respectivement dans les dispositifs Serreslous et Doazit).

Malgré sa mise en œuvre relativement récente (1980), la jachère travaillée est à l'origine d'une chute très sensible du stock carboné ( $7 \mathrm{t} / \mathrm{ha}$ ), inexplicable par la seule interruption des restitu- tions. Une modification profonde des conditions du milieu, et donc des conditions de minéralisation induite par une exploitation différente du sol, est plus vraisemblablement à l'origine de ce phénomène, dont l'intensité souligne le rôle important du mode de gestion des terres en friche sur le devenir de leur statut carboné.

\section{Effet des traitements sur le niveau d'équilibre des contenus en carbone en fonction des régimes de restitution pratiqués}

L'application du modèle exponentiel asymptotique aux traitements TE et TR montre qu'à l'équilibre le différentiel de stock de carbone (valeurs A du tableau $(X)$, entre les parcelles dont on exporte les résidus de culture et celles où on les enfouit, demeure du même ordre de grandeur ( 3 et $5 \mathrm{t} / \mathrm{ha}$ ) que celui observé à la fin de la période expérimentale ( 3 et $4 \mathrm{t} / \mathrm{ha}$ ). En revanche, l'introduction systématique de l'engrais vert se traduit par une tendance à l'abaissement du stock de carbone ( 1 à 4 tha) par comparaison avec les parcelles dans lesquelles on incorpore uniquement les résidus de la culture. Ce constat, à prendre en compte avec une extrême prudence en raison de l'imperfection de la modélisation mathématique tentée, pourrait s'expliquer par l'instauration d'un régime de minéralisation plus intense dans les parcelles engrais vert qui sont soumises à des apports réguliers de matériaux biodégradables.

Comme évoqué précédemment, il n'a pas été possible d'appliquer un tel modèle au traitement PT en raison des ruptures brutales d'évolution au moment des retournements. En conséquence, le taux de carbone à l'équilibre vers lequel pourraient tendre à la longue les parcelles à la fin de chacune des périodes de 4 années de culture de maïs ne peut être quantifié. On notera cependant qu'en 1988, année où aurait dû être mis en place un nouveau cycle de prairie, le différentiel de stock carboné entre les parcelles en alternance maïs-prairie et les parcelles en culture continue était respectivement de 2 et $4 \mathrm{t} /$ ha dans les dispositifs de Doazit et de Serreslous. Ces différences sont du même ordre de grandeur que celles existant au début des cycles antérieurs de prairie, ce qui laisse supposer la persistance à long terme d'un écart moyen de cette ampleur entre les 2 traitements. 


\section{Les limites de la modélisation comme outil de pronostic de l'évolution du statut carboné des sols}

Elles sont clairement mises en évidence par les estimations très différentes de stocks de carbone à l'équilibre auxquelles on parvient selon que l'on utilise le modèle de Hénin et Dupuis (1945) ou un modèle exponentiel asymptotique. Les tableaux VIII et IX montrent en effet que, pour le traitement TR (résidus de récolte enfouis), le niveau d'équilibre oscille en fonction de l'un des 2 modes de calcul choisi, de 13 à 23 tha pour le dispositif Serreslous et de 8 à 31 t/ha dans celui de Doazit. Ces amplitudes considérables sont la résultante des insuffisances des modèles.

\section{Modèle de Hénin et Dupuis (1945)}

Boiffin et al (1986) ont attiré l'attention sur le biais introduit par cette relation qui sous-estime systématiquement le niveau d'équilibre atteint à long terme par le système pour un régime de restitution donné. Cette sous-estimation peut être attribuée à la compartimentation insuffisante du modèle qui ne permet pas de rendre compte de l'existence probable dans le sol de divers types de matière organique possédant des demivies très différentes.

La difficulté, voire l'impossibilité, d'accéder à une estimation conjointe valable des divers paramètres du modèle, même pour une période assez longue (25 années pour le dispositif Doazit), difficulté sans doute expliquable par la très forte corrélation liant $k_{1}$ à $k_{2}$, pose la question de la pertinence même de l'utilisation d'un tel modèle.

L'estimation des 2 coefficients de façon indépendante est également critiquable : l'estimation de $k_{2}$ à partir des données issues des parcelles dont les résidus de récolte sont exportés est biaisée, d'une part, par la non prise en compte des résidus racinaires et, d'autre part, parce que l'on admet implicitement que l'activité biologique est identique dans les parcelles qui reçoivent régulièrement des résidus biodégradables et dans celles qui n'en reçoivent pas. L'évaluation du coefficient $k_{1}$ réalisée en comparant les teneurs résiduelles en carbone des parcelles TR et TE est discutable pour des raisons identiques.

Les limites d'une telle approche apparaissent très clairement quand on estime le coefficient $k_{1}$ relatif au système racinaire en comparant l'évolution des teneurs en carbone dans les parcelles
TE et les parcelles en jachère travaillée (JA). Les valeurs anormalement élevées ainsi obtenues (32 à $60 \%$, sans prendre en compte d'hypothétiques exsudations racinaires), bien que du même ordre de grandeur que celles obtenues de la même manière, et citées par Barber (1979) pour des monocultures de maïs de I'Indiana et de I'lowa (37 et $46 \%$ respectivement), sont en effet difficilement recevables, la composition biochimique des racines n'étant pas suffisamment différente de celle des parties aériennes pour justifier de tels écarts de comportement dans le sol. En fait, ces valeurs représentent un artefact attribuable aux conditions très différentes de minéralisation qui règnent dans les parcelles en jachère travaillée et dans les parcelles portant du maiss, notamment en raison des régimes hydriques distincts qui caractérisent les 2 traitements : durant la période estivale, on observe en effet dans l'horizon de surface de la jachère des teneurs en eau nettement supérieures $(63 \%$ de l'humidité équivalente) à celles ( $25 \%$ de l'humidité équivalente) relevées dans les parcelles voisines portant du maïs (tableau $X$ ) ; les conditions de jachère travaillée, qui s'apparentent en fait à celles caractérisant le "dry-farming", favorisent l'activité microbienne estivale alors que cette dernière est au contraire inhibée dans les parcelles dont les réserves hydriques ont été partiellement épuisées par la culture non irriguée du maïs. Ce phénomène est en accord avec les observations de Jenkinson (1977) qui a montré que la vitesse de disparition d'un ray-grass est beaucoup plus grande quand on l'enfouit en sol nu que sous une prairie asséchant le sol en été ; selon cet auteur, l'accroissement de la richesse en carbone des sols enherbés serait dû en fait autant à une

Tableau $\mathrm{X}$. Teneur en eau de l'horizon labouré en période estivale ( $\mathrm{g} / 100 \mathrm{~g}$ de terre).

Année Mois $\frac{\text { Essai Serreslous }}{\text { TR TE RG JA }} \frac{}{\text { TR TE }}$ TE RG JA

$\begin{array}{llllllll}1986 \text { juillet } 3,5 & 3,3 & 3,8 & 9,3 & 4,0 & 4,0 & 4,7 & 10,0 \\ 1989 \text { juillet } & & & & 7,7 & 8,1 & 8,5 & 10,4 \\ 1989 \text { août } & & & & 5,4 & 5,7 & 6,1 & 10,1 \\ 1990 \text { août } & & & & 4,2 & - & 4,8 & 10,5\end{array}$

TR : parties aériennes restituées ; TE : parties aériennes exportées ; RG : culture intercalaire de ray-grass ; JA : jachère travaillée. 
baisse de l'activité minéralisatrice estivale induite par une sécheresse relative qu'à la production d'une masse importante de résidus organiques.

En résumé, l'évaluation des coefficients $k_{1}$ et $k_{2}$ par une approche expérimentale de terrain n'est concevable que si l'on est certain de l'absolue identité des conditions de minéralisation pour les différents itinéraires techniques ou régimes de restitution organique que l'on doit obligatoirement mettre en œuvre pour conduire cette approche. Comme l'exprime la très forte interdépendance de $k_{1}$ et $k_{2}$, un tel objectif est irréalisable et, en conséquence, la valeur prédictive du modèle qui repose sur ces coefficients se trouve considérablement amoindrie.

\section{Modèle exponentiel asymptotique}

Malgré les écart-types relativement importants affectant certains des paramètres de ce modèle, ce dernier conduit à des valeurs d'équilibre des stocks de carbone (respectivement 23 et 31 t/ha pour les dispositifs Serreslous et Doazit), qui se rapprochent des valeurs effectivement observées (25 tha) sous climat tempéré, après plus de 100 ans de céréaliculture continue, dans l'expérience d'Hoosfield à Rothamsted (Jenkinson et Rayner, 1977). Les valeurs pronostiquées sont également compatibles avec le contenu actuel en matière organique des sols situés à proximité des dispositifs Doazit et Serreslous qui sont soumis depuis plusieurs décennies à la culture continue du maïs.

Néanmoins, malgré la convergence des valeurs d'équilibre fournies par le modèle exponentiel asymptotique avec celles observées à Rothamsted, l'éventualité d'une surestimation ne peut être écartée car ces valeurs sont le résultat de l'extrapolation à long terme du fonctionnement d'un modèle élaboré à partir d'une cinétique contemporaine d'évolution de la teneur en carbone du sol. Les caractéristiques pédoclimatiques du site expérimental (sol de texture grossière, climat doux) ainsi que les très faibles rendements en matière organique stable des résidus de récolte du maïs mis en évidence au cours de cette étude peuvent permettre en effet d'envisager une telle hypothèse.

\section{CONCLUSION}

L'un des points essentiels mis en évidence au cours de cette étude est l'impact limité de régimes de restitution, pourtant quantitativement et qualitativement très distincts, d'une part, sur la cinétique de disparition du carbone et, d'autre part, sur le statut carboné au terme de 2 décennies. Les différences de statut qui s'instaurent après la mise en œuvre de divers régimes de restitution apparaissent comme assez constantes au cours du temps, à l'exception de l'engrais vert pour lequel la modélisation semble pronostiquer l'extinction, à terme, de son effet positif sur le bilan carboné du sol.

Les résultats obtenus confirment par ailleurs les limites d'une modélisation, à caractère statistique et purement descriptif, en tant qu'outil de pronostic à long terme du seuil d'équilibre vers lequel tend la teneur en carbone d'un sol pour un régime de restitution organique donné. Ces limites résultent de la simplicité des modèles proposés, qui se manifeste entre autre par la non prise en compte de paramètres quantifiant les conditions du milieu (température, aération et surtout humidité du sol, importance et fréquence d'incorporation de matériaux biodégradables...) qui régissent prioritairement l'activité minéralisatrice du sol. La chute sensible de la teneur en carbone des parcelles livrées depuis 1980 à la jachère travaillée illustre bien le poids déterminant de ces paramètres en confirmant notamment le rôle essentiel du régime hydrique sur l'évolution du statut carboné du sol.

On doit reconnaître que la rareté de dispositifs de très longue durée situés dans des situations pédo-climatiques différentes représente aussi une difficulté majeure pour l'établissement d'une modélisation prédictive satisfaisante du statut carboné de sols cultivés.

\section{REMERCIEMENTS}

Les auteurs expriment leur vive reconnaissance aux 2 agriculteurs, MM Demen et Laffargue, qui ont bien voulu accepter la mise en place de dispositifs de longue durée sur leurs exploitations. lis adressent également leurs remerciements à $M$ Ciesielski, du laboratoire d'analyses de sols d'Arras, pour son apport très constructif à la partie analytique de l'étude.

\section{RÉFÉRENCES}

Anne $P$ (1945) Sur le dosage rapide du carbone organique des sols. Ann Agron 15, 161-172

Balesdent J, Balabane M (1992) Maize root-derived soil organic carbon estimated by natural ${ }^{13} \mathrm{C}$ abundance. Soil Biol Biochem 24, 97-101 
Barber DA (1979) Corn residue management and soil organic matter. Agron J 71, 625-627

Barber DA, Martin JK (1976) The release of organic substances by cereal roots into soils. New Phytol $76,69-80$

Boiffin J, Keli Zaghabi J, Sebillotte M (1986) Systèmes de culture et statut organique des sols dans le Noyonnais : application du modèle de HeninDupuis. agronomie 6, 437-446

Chadœuf J, Guerif J, Monnier G (1984) Modélisation de l'évolution des matières organiques du sol. In : CR Contrat CEE-INRA n ${ }^{\circ}$ ESE-R-014 F.

Charpentier $P$ (1986) Appréciation du système racinaire chez le maïs. Comparaison de deux méthodes. Mémoire fin d'études ESAP PurpanToulouse, $90 \mathrm{p}$

Delas J, Molot C (1983) Effet de divers amendements organiques sur les rendements du maïs et de la pomme de terre cultivée en sol sableux. agronomie 3, $19-26$

Delphin JE, Conesa A Ph (1979) Évolution de la matière organique du sol d'un essai rotations, irrigation, restitution des pailles dans la plaine de la Hardt. II. Bilan humique. Ann Agron 30, 179-189

Djakovitch JL (1988) Mise au point d'une méthode de détermination rapide du coefficient isohumique de matériaux organiques utilisables pour l'amendement des sols. Thèse CNAM, Bordeaux, $208 p$

Fardeau JC, Guiraud G, Thiery J, Morel C, Boucher B (1988) Taux net annuel de minéralisation de la matière organique des sols de grande culture de Beauce. Conséquence pour l'azote. CR Acad Agric $74,61-70$

Guerif J (1987) Matières organiques et simplification du travail du sol en rotation céréalière. Perspectives Agric 117, 16-19

Hénin S, Dupuis M (1945) Essai de bilan de la matière organique des sols. Ann Agron 15, 17-29

Jenkinson DS (1977) Studies on the decomposition of plant material in soil. $\mathrm{V}$. The effects of plant cover and soil type on the loss of carbon from ${ }^{14} \mathrm{C}$ labelled rye-grass decomposing under field conditions. $J$ Soil Sci 28, 424-434

Jenkinson DS (1988) Soil organic matter and its dynamics. In: Russel's soil conditions and plant growth (A Wild, ed) New York, Longman Scientific and Technical, 11 th ed, $991 p$

Jenkinson DS, Rayner JH (1977) The turnover of soil organic matter in some of the Rothamsted classical experiments. Soil Sci 123, 298-305

Lubet $E$, Juste C (1979) Effet de l'introduction d'une prairie temporaire, d'un engrais vert et de l'exportation des résidus de récolte sur les monocultures de maïs implantées dans les sols sablo-limoneux du sud des Landes. CR Acad Agric Fr 65, 295-309

Lubet $E$, Juste $C$ (1985) Cinétique de la production de matière sèche et des prélèvements d'éléments nutritifs par une culture irriguée de maïs à haute potentialité de rendement. agronomie 5, 239-252

Lubet $E$, Plénet $D$, Juste $C$ (1993) Effet à long terme de la monoculture sur le rendement en grain du maïs (Zea mays L) en conditions non irriguées. agronomie 8, 673-683

Müller JC (1982) Matière organique : à quel niveau la maintenir ? Cultivar 1, 60-64

Müller JC, Rémy JC (1980) Influence de l'azote minéral sur la cinétique de décomposition des pailles. In: CR Contrat CEE-INRA n ${ }^{\circ}$ 326-78-1 ESF

Picard D (1983) Influence de l'enfouissement répété des tiges. Agromaïs 19, 16-18

Rémy JC, Marin-Laflèche A (1976) L'entretien organique des terres. Coût d'une politique de l'humus. Entrepr Agric 11, 63-67

Sauerbeck DR, Johnen BG (1977) Root formation and decomposition during plant growth. In: Soil organic matter studies. IAEA, Vienne, $424 \mathrm{p}$

Tardieu F, Manichon G (1987) État structural, enracinement et alimentation hydrique du maïs. II. Croissance et disposition spatiale du système racinaire. agronomie $7,201-211$ 